Int. J. Dev. Biol. 58: 379-384 (2014)

doi: $10.1387 / \mathrm{ijdb} .140024 \mathrm{jt}$

\title{
Analysis of NUAK1 and NUAK2 expression during early chick development reveals specific patterns in the developing head
}

\author{
ABDELHAMID BEKRI\#, MARC BILLAUD and JACQUES THÉLU*
}

Univ. Grenoble Alpes, INSERM, CNRS, France

\begin{abstract}
Several human diseases are associated with the NUAK1 and NUAK2 genes. These genes encode kinases, members of the AMPK-related kinases (ARK) gene family. Both NUAK1 and NUAK2 are known targets of the serine threonine kinase LKB1, a tumor suppressor involved in regulating cell polarity. While much is known about their functions in disease, their expression pattern in normal development has not been extensively studied. Here, we present the expression patterns for NUAK1 and NUAK2 in the chick during early-stage embryogenesis, until day 3 (Hamburger and Hamilton stage HH20). Several embryonic structures, in particular the nascent head, showed distinct expression levels. NUAK1 expression was first detected at stage $\mathrm{HH} 6$ in the rostral neural folds. It was then expressed (HH7-11) throughout the encephalalon, predominantly in the telencephalon and mesencephalon. NUAK1 expression was also detected in the splanchnic endoderm area at HH8-10, and in the vitellin vein derived from this area, but not in the heart. NUAK2 expression was first detected at stage $\mathrm{HH} 6$ in the neural folds. It was then found throughout the encephalon at stage HH20. Particular attention was paid in this study to the dorsal ectoderm at stages HH7 and HH8, where a local deficit or accumulation of NUAK2 mRNA were found to correlate with the direction of curvature of the neural plate. This is the first description of NUAK1 and NUAK2 expression patterns in the chick during early development; it reveals non-identical expression profiles for both genes in neural development.
\end{abstract}

KEY WORDS: NUAK, chick embryo, in situ hybridization

NUAK1 and NUAK2 are members of the novel (Nua) kinases family. Their alternative names include: ARK5 or OMPHK1 for NUAK1; and SNARK or OMPHK2 for NUAK2. They are two of the fourteen known substrates of the serine threonine kinase LKB1 and are part of the AMPK-related protein kinase family (ARK)(Al-Hakim et al., 2005, Hardie and Alessi, 2013, Manning et al., 2002, Suzuki et al., 2003). AMPK is a mammalian homolog of sucrose non-fermentable protein kinase (SNF-1); it is a member of a serine/threonine protein kinase family. Unlike AMPK, which is activated under various stress conditions resulting in decreased ATP concentrations (Scott et al., 2007), ARKs do not have AMP: ATP ratio-sensitive regulatory subunits. Thus, they do not directly participate in adaptive responses to energy balance (Al-Hakim et al., 2005).

NUAK1 was first described as supporting AKT-dependent cell survival during nutrient starvation (Suzuki et al., 2003), and has been shown to induce p53 phosphorylation (Hou et al., 2011). NUAK1 expression is now known to be closely associated with metastases, colorectal cancer and is an indicator of poor progno- sis for glioma (Lu et al., 2013). Recently, it was shown to favor an invasive phenotype in human breast cancer which also depended on AKT signaling. This may be linked to its role in cell adhesion through phosphorylation of the regulatory sub-unit of the myosin light chain 2 (MLC2) phosphatase complex. Other roles for NUAK1 include: critical role in neurodegenerative diseases; maintenance of metabolic homeostasis; limiting protein synthesis via the mTORC1 signaling pathway; regulating ploidy and senescence via LATS1 kinase. For a general review about regulation and function of the NUAK family see (Sun et al., 2013)

Together, NUAK1 and NUAK2 inhibit MLC2 phosphatase through three entry points. This complex regulation of MLC2 phosphorylation strictly controls actin stress fiber assembly and contractility,

Abbreviations used in this paper: ARK, AMPK-related kinase; $\mathrm{HH}$, Hamburger and Hamilton stage; ISH, in situ hybridization; MLC2, myosin light chain 2; NCC, neural crest cell; NLS, nuclear localization signal; NT, neural tube; PFA, paraformaldehyde.

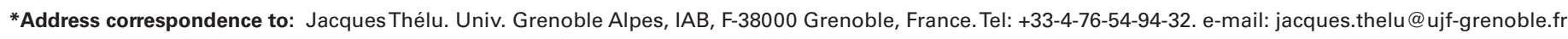
"Present address: Département de Neurosciences, Centre de Recherche du Centre Hospitalier de I'Université de Montréal (CRCHUM), Montréal, Canada.

Supplementary Material (2 figures) for this paper is available at: http://dx.doi.org/10.1387/ijdb.140024jt

Accepted: 10 April 2014. Final, author-corrected PDF published online: 30 September 2014.

ISSN: Online 1696-3547, Print 0214-6282

(C) 2014 UBC Press

Printed in Spain 
NUAR1 MEGAEA_AACGSSSSPAQPRCSSGGEA_ARAAMEEPLGEAASPGVAATAAGPRKQQGVKRHH 60

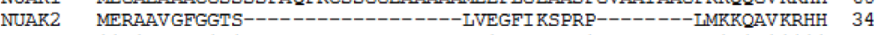
$* * * \ldots *: * \quad: *::: * * \quad+* * * * * *$

NUAR1 HKHNLRHRYELQETLGKGTYGKVKRAIERFSGRVVÄIKSIRKDKIKDEQDMVHIRREIEI 120 NUAK2 HKHNLKHRYEFLETLGKGTYGKVKKARER-SGKLVAIKSIRKDRIKDEODLIHIRREIEI 93

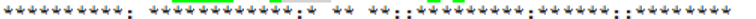

NUARI MSSLSHPHIITIYEVFENKDKIVIIMEYASKGEIYDYISERRRLSERETRHFFRQIVSAV 180 NUAR2 MSSLNH PHIIAVHEVFENSSKIVIVMEYASKGDIYDYISERQRLSEQEARHFFRQVVSAV 153

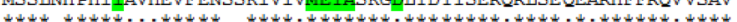

HYCHKNGVVHRDIKLENILIDDNFNI KIADFGLSNLYHKDKFLOTFCGSPLYASPE IVNG 240 NUAR2 YYCHKNGIVHRDIRLENILIDANGNI RIADFGLSNVYQQDKFLCTYCGSPLYASPEIING 213 :

NUAR1 RPYRGPEVDSWLGVLIYTLVYGTMP FDGFDHKNLIRQISSGEYRE PTQTSDARGL IRWM 300 NUAR2 RPYKGPEVDSWSLGVLIYILVHGTMP FDGHDYKTLVROITSGDYRE PTKLSDACGLIRWM 273

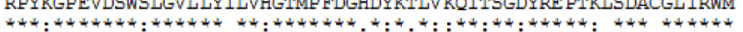

NUAK1 LMVNPERRATIEDIANHWWVNWGYKSSVCDCDAMRDSESPLLARFIDWHHRSTGLQPETD 360 NUAR2 LMVNPERRATIEDIATHWWVNWGYRV PVGEOELIRESSESPIATVAEWLRRSSRPL FENG 333

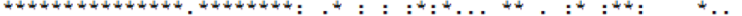

NUAR1 TKMRCLSKPKGPEVTLERQRSLKKSKKENDIAQSIQEGGA--ENASKPTSKRPKGILKKR 420 NUAK2 SKVRCFLKOHIPGVALERORSLKKSK KENDVSHALOEGAARPENPS KSILKRPKGILKKR 393

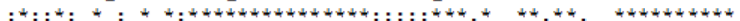

NUAK1 SNSEHRSHSAGFIEGVVSPVLPSAFKLEOELCRTGVAIKTVVEGEVAGKYGTKOSSIMPK 480

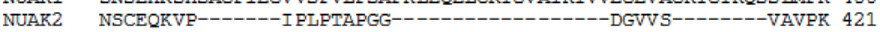

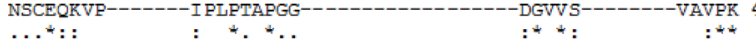

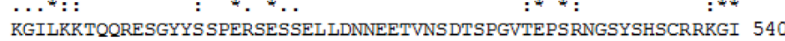
KGILKK PPKRESGYYSSLECCESGDVLDAGSLDLEGNVFADSPTAER-GPPVLPSSRKGI 480

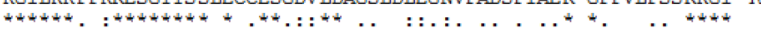

NUAR1 LKHNGKYSTSSTESALISPDTPTLEAMEEVVLPGDALPRSYSRPSSVISDDSILSSDSFD 600 NUAR2 LKHSSKYTSG---AEPHSPPRQSFGCFDEVSLPKAPLAP-RPRPPSAVSEDSILSTESFE 536

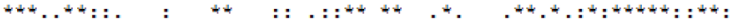

NUAR1 LLDLOENRPNRQR--IRSCVSAENFLQIQDFEGLQNHPRPQ--YLKRYRNRLGDSSFSLL 660 NUAR2 QLDLPFRVPGRSGMMRGCVSADSLILEEEEEVDEGRRLRRWTVTHCPTALGESRFSLE 596

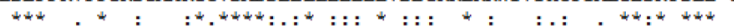

NUAK1 ADMDDVTQVYKKALEICNKLN 671

NUAR2 S-CDNVTEVHRRAVAISMRLS 615

S-CDNVTEVHRRAVAISMKLS
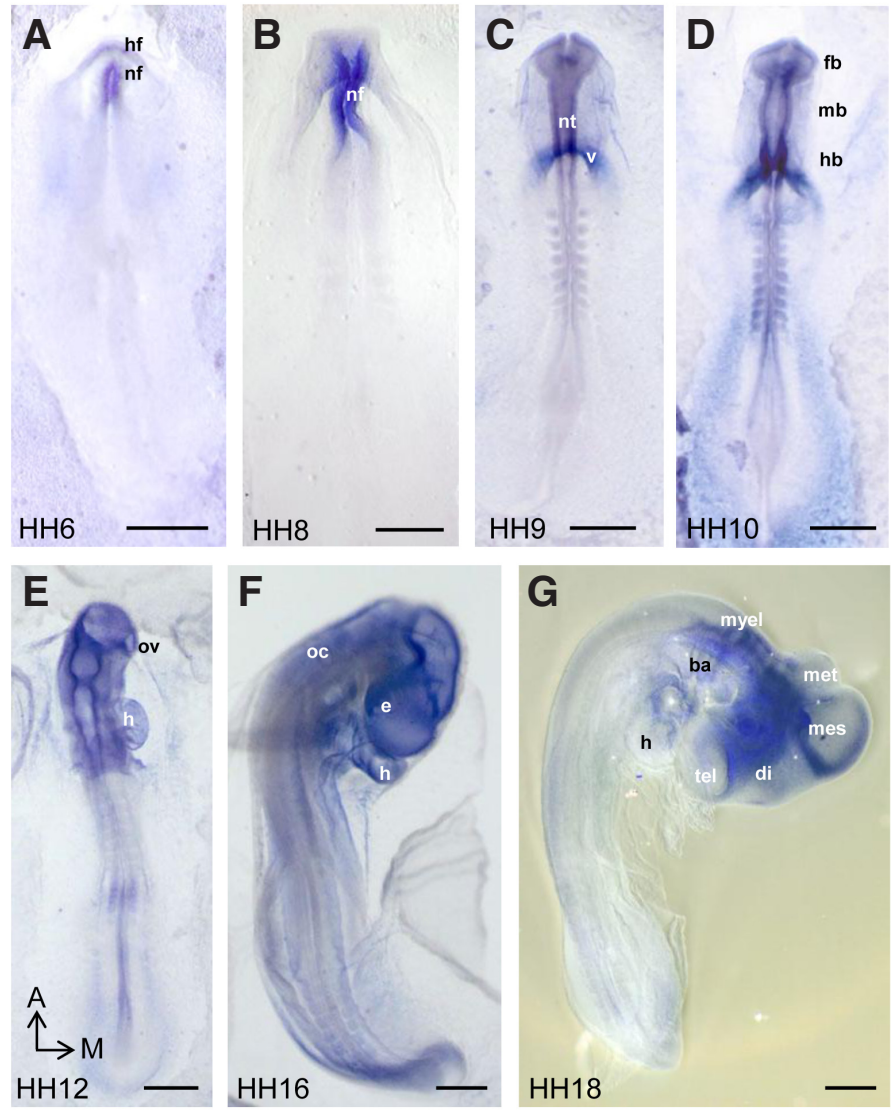

Fig. 1 (left). Amino acid sequence alignment for Gallus gallus as NUAK1 "XP_416310.2" and NUAK2 "XP_417962.2". Positions with a single, fully conserved residue (52.9\%) are indicated by an asterisk (*); conservative substitutions are indicated by dots under the sequences. Two dots (:) (15.0\%) indicates conservative substitution within a strong amino acid group, score greater than 0.5 ; one dot (.) (8.4\%) indicates conservative substitution within a weak group, score 0.5 or less. The ATP binding sites in the NUAK catalytic domain are shown in green and blue, the domains of NUAK1 and NUAK2 activated by LKB1 phosphorylation (T loops) are shown in yellow and blue, with the phosphorylation sites (Thr ${ }^{225}$, Thr ${ }^{198}$ and Ser ${ }^{229}$, Ser ${ }^{202}$ ) shown in black. The conserved ${ }^{58} K K A R^{61}$ sequence which corresponds to the NUAK2 nuclear localization signal (NLS) is indicated in grey. The conserved ${ }^{611} R Q R I R S^{616}$ AKT phosphorylation motif is indicated in red, with the putative phosphorylation site, Ser6i6, shown in black.

Fig. 2 (Right). NUAK1 expression pattern in HH6 to HH18 chick embryos. Whole-mount embryo images were viewed dorsally (A-E) and laterally (F,G). Abbreviations: A-M, anterior-medial; ba, branchial arch; di, diencephalon; e, eye; fb, forebrain or prosencephalon; $h f$, head fold; $h b$, hindbrain or rhombencephalon; h, heart; mb, midbrain or mesencephalon; mes, mesencephalon; met, metencephalon; myel, myelencephalon; nf, neural folds; nt, neural tube; oc, otic cup; ov, optic vesicle; tel, telencephalon; v, vitelline vein. Scale bar, 500um.

as well as cell polarity, motility and invasiveness.

In line with this function, NUAK2 locates along actin filaments in cultured non-muscle cells (Vallenius et al., 2011). Previous work had shown that NUAK2 was predominantly located in the nucleus in HeLa cells (Kuga et al., 2008), which was expected given its conserved $\mathrm{N}$-terminal nuclear localization signal $\left({ }^{68} \mathrm{KKAR}{ }^{71}\right.$ in chick, Fig. 1). In terms of human disease, NUAK2 was shown to contribute to the development of melanomas (Namiki et al., 2011). Other roles for NUAK2 were identified in heterozygous NUAK2 mice, which display mature-onset obesity and related metabolic disorders and also have a higher risk of developing colorectal cancers. In skeletal muscle, NUAK2 mediates glucose transport and its expression is regulated by metabolic stress and diabetes. Thus, this AMPK-related kinase is thought to be a regulator of whole-body metabolism (Egan and Zierath, 2009, Sun et al., 2013).

Despite this wealth of potential roles and activities, the normal in vivo functions of NUAK1 and NUAK2 have not yet been fully elucidated. Although these genes are mainly expressed in the neuroectoderm in mouse, it has been reported that NUAK1 mutants exhibit no apparent gross brain defects, but do have ventral body wall defects (Hirano et al., 2006). Mice deficient in both NUAK1 and NUAK2 display exencephaly, facial clefting, and spina bifida, suggesting partially redundant activity for these two genes (Ohmura et al., 2012). In addition, in these mice, phosphorylated MLC2, F-actin and cortactin fail to effectively concentrate at apical cell surfaces, indicating that NUAK kinases may contribute to apical constriction and cell shape control. NUAK1 and NUAK2 functions have been studied in human (Lu et al., 2013, Sun et al., 2013), mouse (Ohmura et al., 2012), D. melanogaster (Amin et al., 2009) and C. elegans (Hoppe et al., 2010). In this article, we report on NUAK1 and NUAK2 mRNA expression in early chick embryos. This model is frequently used to study neurulation, neural crest migration and head formation, all of which may require activity of these two genes. Through in situ hybridization of specific mRNA 
probes and imaging of transverse sections, we had detected that expression of NUAK1 is most prominent throughout the neuroectoderm and that the pattern for NUAK2 expression suggests a role in remodeling epithelial tissue during head formation.

\section{Results and Discussion}

\section{Amino acid sequence alignment}

Comparisons of chick NUAK1 and NUAK2 amino acid sequences with homologs from other species reveals high levels of sequence conservation: $80.3 \%$ and $64.2 \%$ similarity to human sequences and $52.7 \%$ and $61.4 \%$ similarity to mouse sequence, respectively (Supplementary Fig. S1). Chick NUAK1 and NUAK2 are also similar to one another, with an identity score of $52.9 \%$, and $15.0 \%$ substitution with a strong degree of conservation (Fig. 1). Like AMPK and other ARKs (except MELK), chick NUAK1 and NUAK2 can be phosphorylated by LKB1 on conserved threonine and serine residues ( $\mathrm{Thr}^{225}$, and $\mathrm{Ser}^{229}$ on chick NUAK1; and Thr ${ }^{198}$ and $\mathrm{Ser}^{202}$ on chick NUAK2; highlighted in black, Fig. 1). These residues are part of the conserved T-loop sequences (Kuga et al., 2008) (indicated in yellow and blue, in Fig. 1). The ATP binding sites in the NUAK catalytic domain are shown in green and blue in Fig. 1. Unlike NUAK1, NUAK2 is believed to locate to the nucleus (Kuga et al., 2008). This localization is supported by the consensus KKAR nuclear localization signal (NLS) at position 58-

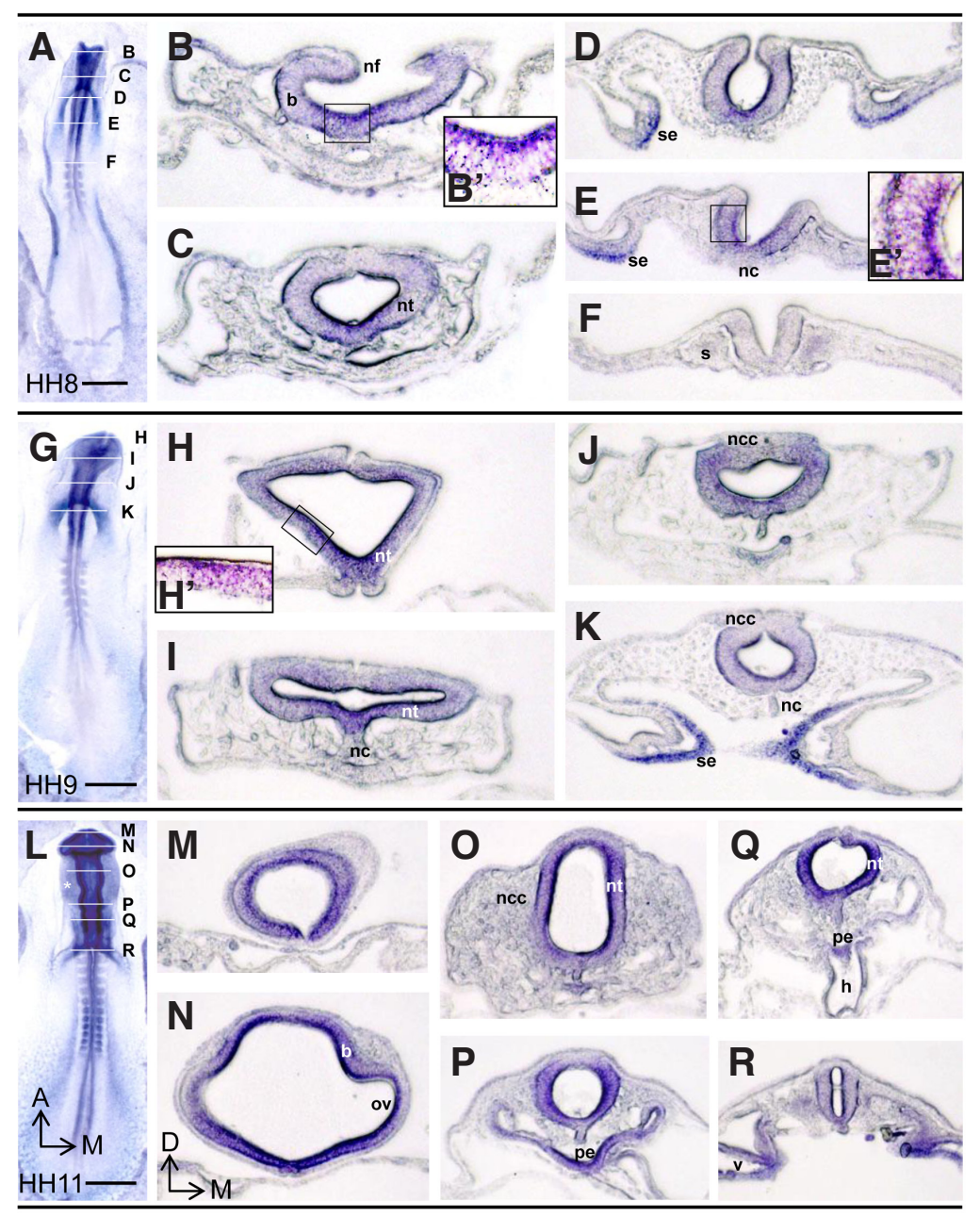

61 (indicated in grey, Fig. 1). Conversely, NUAK1, but not NUAK2, has a RQRIRS AKT phosphorylation site at position 611-616 in the chick sequence (shown in red, Fig. 1). Evidence suggests that phosphorylation at this site results in protein activation (Suzuki et al., 2003).

\section{NUAK1 gene expression}

During chick development, NUAK1 expression was not detected before stage HH6. At this stage, expression was observed in the neural folds (nf), and in the head fold (hf); the primitive streak and Hensen's node were negative (Fig. 2A). Expression increased with development from the head fold stage to head formation (Fig. 2 $B-G)$, with strong expression in the hindbrain region at $\mathrm{HH} 10$ (Fig. 2D). Sections at stages HH8-11 show strong expression in the cephalic neural tube (Fig. 3). At stage $\mathrm{HH} 8$ in the head forming position, when the neural ridges meet and are beginning to fuse, NUAK1 was mainly expressed at apical side of epithelial cells located on NT floor (Fig. 3 B-D,B'). The neuroectoderm is a pseudo epithelium with a majority of cells going from basal to apical side, then NUAK1 mRNA are polarized to the apical pole in these cells. At a more caudal level, where the neural folds splay out, NUAK1 was mainly expressed at the apical side of all the cells in the groove's walls (Fig. 3E,E'). From the level of the first somite (Fig. $3 \mathrm{~F}$ ), until the caudal primitive fold, no NUAK1 mRNA was detected in neuroectoderm. At stage $\mathrm{HH}$, in rostral position, NUAK1 was present in neuroectodermal cells, with a slightly higher level at the apical side. In this position, NUAK1 expression was at low level at roof side (Fig. $3 \mathrm{H}-\mathrm{K}, \mathrm{H}^{\prime}$ ). Labeling was decreased at a more caudal level (Fig. $3 \mathrm{~K}$ ). The thin black lines in Fig. 3 correspond to shadows due to the thickness of slices. At stage H11, NUAK1 expression was reinforced (Fig. $3 \mathrm{M}-\mathrm{Q}$ ). Neural crest cells (ncc), which migrate laterally from the point where the dorsal neural folds meet, presented only faint or no NUAK1 expression in whole-mounts (Fig. $3 \mathrm{C}, \mathrm{D}, \mathrm{H}-\mathrm{K}, \mathrm{N}-\mathrm{Q}$, asterisk in Fig. 3L). However, we observed a low NUAK1 expression at somites level (Fig. 3A,F,G,L). The notochord (nc) showed almost no expression (Fig. 3 B-F,I) or only weak expression (Fig. $3 \mathrm{~J}, \mathrm{O}, \mathrm{R}$ ). The extra-embryonic regions, area pellucida and area opaca, showed no expression of this gene (Fig. 2 A-D). In contrast, expression was high in the region surrounding the nascent vitellin veins (v) in wholemount embryos (Fig. 2 C,D), and in sections showing the splanchnic endoderm (Fig. $3 \mathrm{D}, \mathrm{E}, \mathrm{K}$ ). Other endodermal tissue also expressed high levels of NUAK1, e.g. in the pharynx (Fig. 3P). In the nascent heart, staining was faint at $\mathrm{HH} 11$ (Fig. $3 \mathrm{P}, \mathrm{Q}$ ), HH12 (Fig. 2E) and HH16 (Fig. 2F) and was no longer detected at HH18 (Fig. 2G).

Fig. 3. NUAK1 expression pattern in HH8 to HH11 chick embryos. The position of each section is indicated by white lines in the whole-embryo images $(\mathbf{A}, \mathbf{G}, \mathbf{L})$. $\left(\mathbf{B}^{\prime}, \mathbf{E}^{\prime}, \mathbf{H}^{\prime}\right)$ are zooms of the boxed areas in $(\mathbf{B}, \mathbf{E}, \mathbf{H})$ showing specific NUAK1 expression in the neuroectoderm of the head. Migrating NCC express low levels of NUAK1 in lateral positions (asterisk in L,O). Abbreviations: A-M, anterior-medial; D-M, dorsal-medial; b, brain; $h$, primordial heart; $n c$, notochord; ncc, neural crest cells; nf, neural folds; nt, neural tube; ov, optic vesicle; pe, pharyngeal endoderm; s, somite; se, splanchnic endoderm; v, vitelline vein. Scale bar, 500um. 


\section{NUAK2 gene expression}

NUAK2 expression was first detected in the rostral region at stage $\mathrm{HH} 6$, in the dorsal neural folds and in the head fold region (Fig. 4A). Almost no expression was detected in the primitive streak or Hensen's node itself. In contrast to NUAK1, NUAK2 was extensively expressed in the lateral extra-embryonic domain (Fig. 4 A-D). At stages $\mathrm{HH} 7$ to $\mathrm{HH} 9$, NUAK2 expression increased in the nascent head and in the spinal cord, while faint

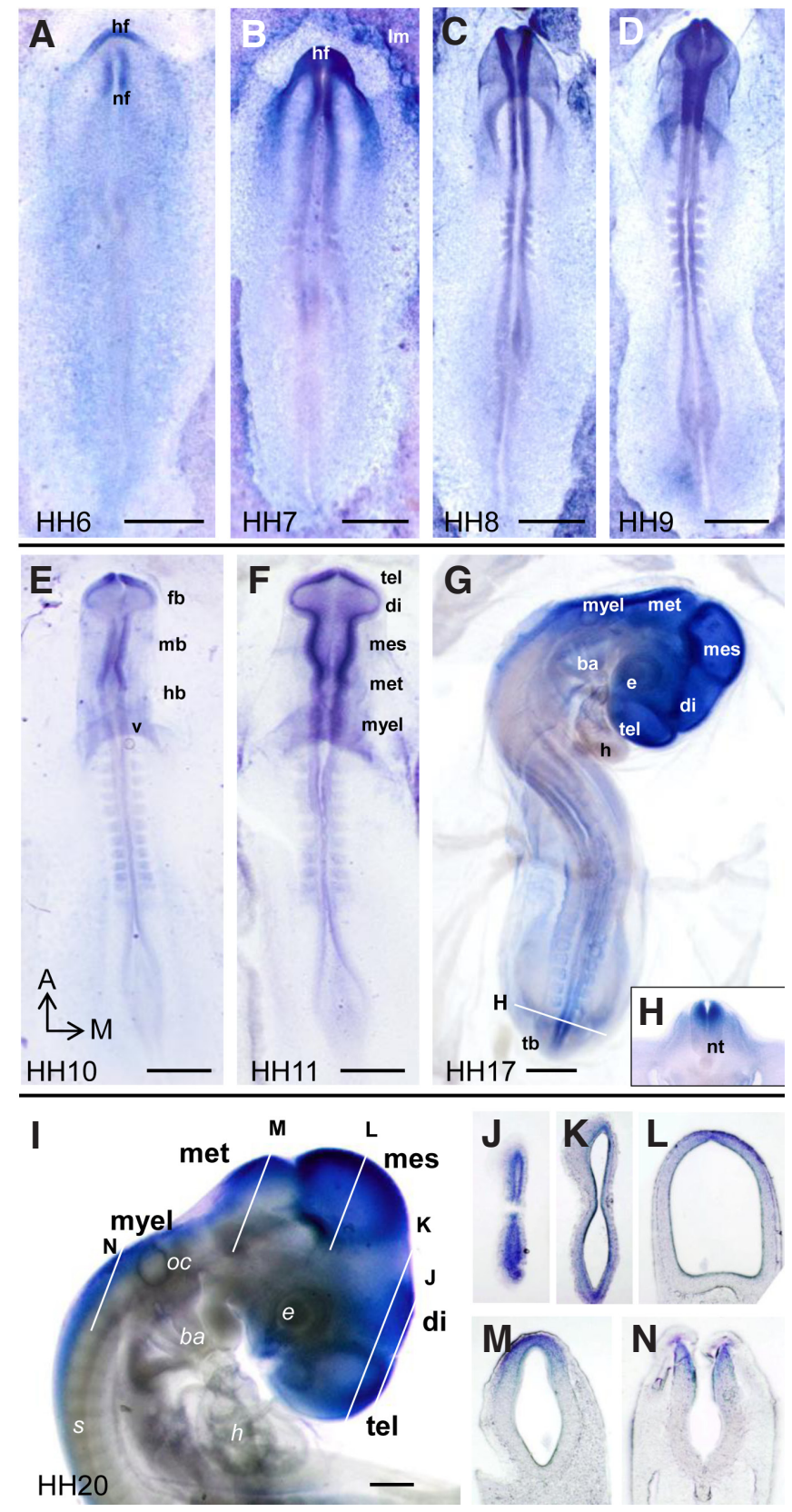

Fig. 4. NUAK2 expression pattern in $\mathrm{HH} 6$ to $\mathrm{HH} 17$ chick embryos. The position of each section is indicated by white lines in the images $(\mathbf{G}, \mathbf{I})$. Abbreviations: A-M, anterior-medial; ba, branchial arch; di, diencephalon; $e$, eye; fb, forebrain or prosencephalon; $h$, heart; $h b$, hindbrain or rhombencephalon; hf, head fold; Im, lateral plate mesoderm; mb, midbrain or mesencephalon; mes, mesencephalon; met, metencephalon; myel, myelencephalon; $n f$, neural folds; nt, neural tube; oc, otic cup; s, somite; th, tail bud; tel, telencephalon; v, vitelline vein. Scale bar, 500um. labeling was observed in somites (Fig. 4 B-D). At stages $\mathrm{HH} 10$ and $\mathrm{HH} 11$, neural expression faded except in the telencephalon, the mesencephalon and the rostral half of the metencephalon (Fig. 4 E,F). Very faint expression could be seen in the caudal NT region and in somites (Fig. 4 E,F). At stage $\mathrm{HH} 17$, expression was found throughout the encephalon, the eye, the otic cup (Fig. 4G) and in the dorsal NT of the caudal region in transverse sections (Fig. 4H). At stage $\mathrm{HH} 20$, expression was restricted to the encephalon, as seen in whole-mounts (Fig. 4I) and sections (Fig. $4 \mathrm{~J}-\mathrm{N}$ ). These sections showed expression to be restricted to the dorsal part of the central nervous system (CNS) this dorsal staining was consistent with that observed in the caudal NT (Fig. 4H). The most anterior section (Fig. 4J) with no cavity shows a strong NUAK2 expression, this allows us to exclude a potential artifact related to the trapping of purple precipitate in cavities. To definitively exclude this possibility, we also performed negative and positive controls (Supplementary Fig. S2).

Particular attention was paid to NUAK2 expression in the ectoderm at stages $\mathrm{HH} 7$ and $\mathrm{HH} 8$ (Fig. 5). During neural groove and head formation, several hinges form successively by curvature of the ectodermic epithelium. The NUAK2 expression level seems to match this curvature. The first fold is created at the primitive groove (Fig. 5M). NUAK2 expression was low at the concave medial hinge point $(\mathrm{mh})$ of this fold (black asterisk Fig. 5L) compared to the posterior-most position (Fig. $5 \mathrm{~N}$ ) of the developing embryo, where NUAK2 is expressed in a roughly flat ectoderm. Fig. 5L showed two additional convex dorsal hinges (dh) with high levels of NUAK2 mRNA in ectoderm (white asterisk). The intensity of expression and the variation between apico-basal positions in the ectoderm were also observed in other images, especially at positions where the epithelium curves (arrowheads Fig. 5 B'-E'). The convex dh and lateral hinge (Ih) at cranial level (Fig. $5 \mathrm{C}, \mathrm{G}, \mathrm{H}$ ) presented high levels of expression. On the other hand, no NUAK2 expression was detected in the neuroectoderm between the $\mathrm{mh}$ and the $\mathrm{dh}$ (Fig. 5 B-E,G). The boundary between positive and negative domains located in the neural crest territory (white asterisk in Fig. $5 \mathrm{H}$ ) is clearly observable (black dashed line Fig. $5 \mathrm{H}$ ). The mesoderm in axial position, which underlines the medial hinge, expresses NUAK2 (Fig. 5 C-E).

\section{Conclusion}

In this paper, we describe the expression patterns for NUAK1 and NUAK2during early chick embryogenesis. We also performed bioinformatics analysis to determine sequence conservation between these two homologs. The expression patterns we describe are similar to those previously obtained for NUAK1 in wild-type mice embryos using a $\beta$ Gal fusion protein (Hirano et al., 2006). In the chick, the regionally-restricted expression of NUAK isoforms overlaps. Both genes are expressed in the rostral neural folds during head formation, and only very low NUAK1 and NUAK2 mRNA expression was detected at the confluence of the neural folds, from where NCC originate. However, specificities did emerge as NUAK2 was transcribed in the extra-embryonic regions and in the ectoderm of the forming head, while NUAK1 was not. Conversely, NUAK1 was transcribed in the neural tube and NUAK2 was not. At later stages, from $\mathrm{HH} 17$ to $\mathrm{HH} 20, N U A K 1$ and NUAK2 have similar expression patterns, largely focused in the brain. This observation leads us to suggest that these two genes have distinct and complementary roles over the course of head development. 

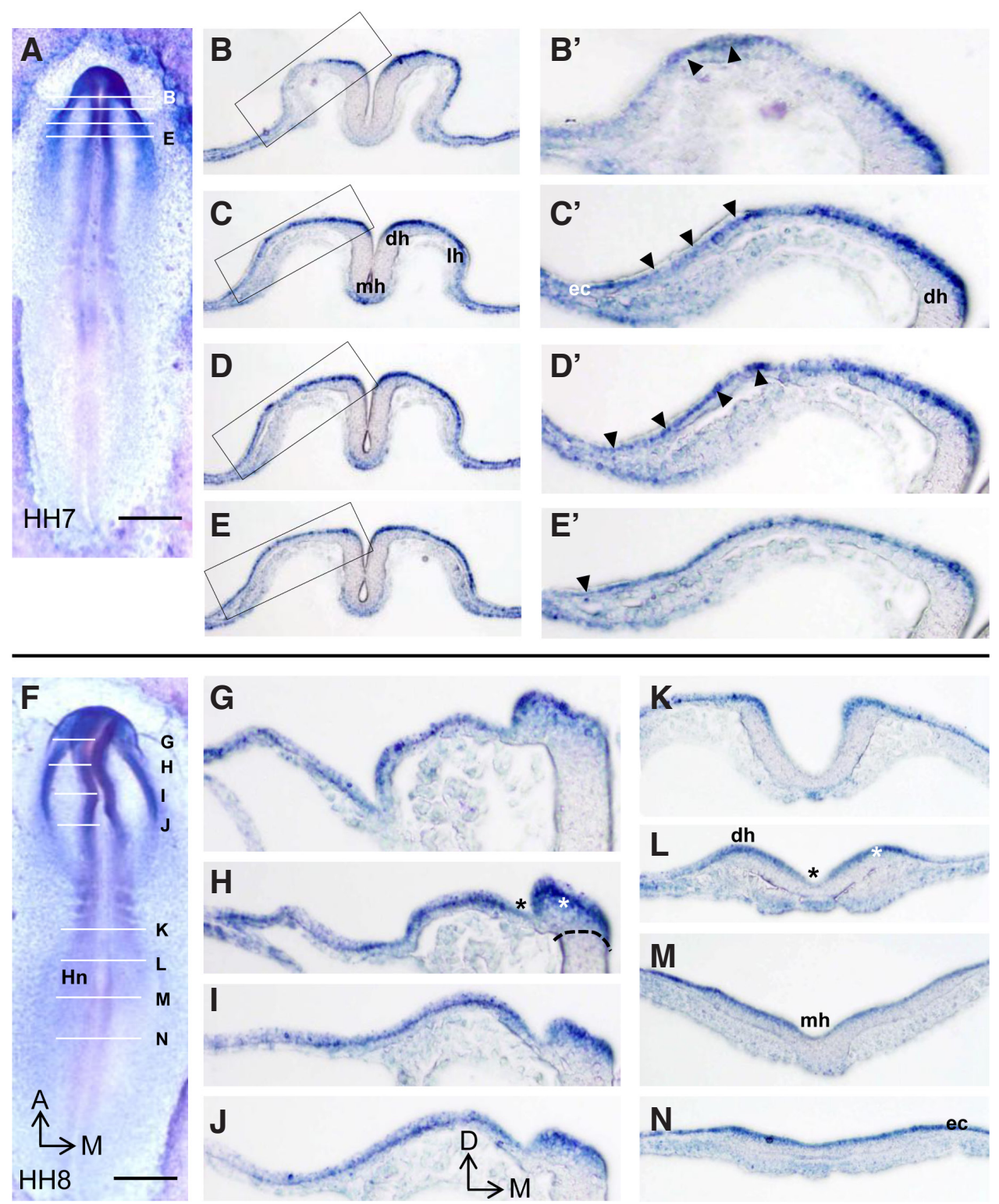

Fig. 5. NUAK2 expression at HH7 and HH8 chick embryos. (B-E) Sections indicated in (A) by white lines. (B' - E') Zooms of the boxed areas in (B - E). (G -N) Sections indicated in (F) by white lines. Black arrow heads indicate variations in the position of NUAK2 mRNA staining in the ectoderm. Black asterisks indicate low NUAK2 mRNA levels in ectoderm; white asterisks indicate high NUAK2 mRNA levels

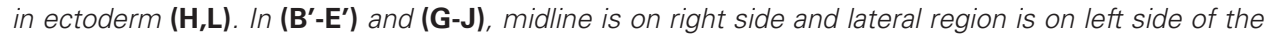
images. (H) The positive/negative boundary is underlined with a black dashed line. Abbreviations: A-M, anterior-medial; D-M, dorsal-medial; ec, ectoderm; dh, dorsal hinge point; Hn, Hensen's node; Ih, lateral hinge point; mh, median hinge point. Scale bar, $500 \mu \mathrm{m}$.

This hypothesis is supported by the phenotype of double knockdown mice (Ohmura et al., 2012). Ohmura et al., concluded that NUAK1 and NUAK2 have complementary functions in the apical constriction and apico-basal elongation associated with dorsal hinge point formation in the cephalic neural plate during the 5 - to 10-somite stages. Interestingly, LKB1, which phosphorylates both NUAK homologs, was shown to regulate changes in polarity and adherens junction formation in the Drosophila eye (Amin et al., 2008). In addition, ARK CG11871 gene (Drosophila homolog to mammalian NUAK) RNAi experiments revealed developmental defects that partially phenocopy $l k b 1$ mutants. Altogether, these observations are consistent with a link between the LKB1/NUAK pathway and cell shape. In support of this, we observed a lack or an accumulation of NUAK2mRNAin the ectoderm at stages $\mathrm{HH} 7-8$, which correlated with concave or convex neural plate curvature. Furthermore, the bending profile of the cephalic ectoderm matches to NUAK2 mRNA detection in epithelial structures. These observations are clues pointing to a role for NUAK2 in morphogenesis according to Ohmura et al., (2012). Interestingly, regulation of NUAK2 expression by a dorsally-derived morphogene (BMPs or Wnts) might also give this type of staining. Furthermore, the expression pattern shown in Fig. $5 \mathrm{~K}-\mathrm{N}$ suggests repression of the expression of NUAK2 by a neurogenesis-related ventral signal, such as Shh (for a review see Ulloa and Marti, 2010).

\section{Materials and Methods}

\section{Sequence analysis}

The Gallus gallus NUAK protein sequences were extracted from the NCBI database (http://www.ncbi.nlm.nih.gov) as NUAK1 "XP_416310.2" and NUAK2 "XP_417962.2". Sequence alignment and amino acid identity analysis was performed using the ClustalW algorithm (http://www.clustal.org). The positions of putative specific domains were taken from the literature: signature protein kinase ATP-binding regions and LKB1 phosphorylation sites for NUAK1 (Suzuki et al., 2003) and NUAK2 (Lefebvre et al., 2001); NUAK1-AKT phosphorylation motif (Suzuki et al., 2003); NUAK2-nuclear localization signal (NLS) (Kuga et al., 2008).

\section{Isolation and cloning of NUAK1 and NUAK2 probes}

Total RNA was isolated from E2 total embryo extracts from chick strain JA957. RNA was reverse transcribed to produce cDNA. Sequence-specific nested primer sets (Table 1) were used to clone partial sequences for both genes (NUAK1 "Gene ID: 418074" and NUAK2 "Gene ID: 419828"). An 810 bp PCR product for NUAK1 and an 830 bp PCR product for NUAK2 were cloned in TA cloning pGEM-TEasy vectors (Promega). Clones were sequenced using the M13 primer (Beckman Coulter Cogenics). To produce antisense RNA probes, NUAK1 plasmids were digested with Nco I and transcribed with Sp6 RNA polymerase; NUAK2 plasmids were digested with Spe I and transcribed with T7 RNA polymerase and NUAK2 plasmids were digested with Apa I and transcribed with Sp6 RNA polymerase to produce a control sense probe. Digoxygenin (DIG)-labeled RNA antisense and sense probes were transcribed using Roche reagents, according to the manufacturer's instructions. FGF8 plasmids were digested with Eco RI and transcribed with T7 RNA polymerase to synthetize an antisense RNA probe used as a positive control (Supplementary Fig. S2). 
TABLE 1

\section{NESTED PRIMER SETS USED TO CLONE CHICK NUAK1 AND NUAK2 PROBES}

\begin{tabular}{llll} 
& & Forward primers (5'- $\mathbf{3})$ & Reverse primers $\mathbf{( 5}$ ' $\mathbf{3}$ ') \\
\hline NUAK1 & Outer set & GCGGCATCACCACAAGCACA & GTGATGCCAATCAATGAACC \\
& Inner set & CTGCAGGAGACCTTGGGCAA & TGGAGACTCAGAGTCCCTCA \\
NUAK2 & Outer set & CATCGCTACGAGTTCCTGGA & CAAGAAGCACCTCACTTTGG \\
& Inner set & ATGGGAAGTGAAGAAAGA & AAAGAGTGGTCGGGGGAGC \\
\hline
\end{tabular}

\section{Embryos and in situ hybridization}

Fertilized chick eggs (JA957 strain, SFPA, St Marcellin, France) were incubated at $38^{\circ} \mathrm{C}$ in a humidified incubator, until embryos reached stage HH6-20, according to the Hamburger and Hamilton table (Hamburger and Hamilton, 1951). Embryos were immediately fixed in ice cold $4 \%$ paraformaldehyde (PFA) solution in PBS. They were then dehydrated in a series of methanol baths ( $25 \%, 50 \%, 75 \%$, methanol in PBS), followed by $100 \%$ methanol before storing them at $-20^{\circ} \mathrm{C}$. The whole-mount in situ hybridization protocol was adapted from Wilkinson and Nieto (Wilkinson and Nieto, 1993). Once rehydrated, embryos were loaded into sample holders and subjected to a continuous stream $(1.7 \mathrm{ml} / \mathrm{min})$ of successive reagents in an automaton (Flogentec, France) as follows. Samples were washed (15 min) with PBS and $0.1 \%$ tween 20 (PBT), then treated with proteinase $\mathrm{K}(20 \mu \mathrm{g} /$ $\mathrm{ml}$ ) in PBT at room temperature (5 to $20 \mathrm{~min}$, depending on embryo size). After washing for $15 \mathrm{~min}$, embryos were post-fixed with 4\% PFA solution for $20 \mathrm{~min}$, then washed once again for $15 \mathrm{~min}$. Samples were exposed to hybridization mix (50\% formamide; $5 x \mathrm{SSC} ; \mathrm{pH}=4.5 ; 0.1 \%$ tween 20 ) for 1 hour at $70{ }^{\circ} \mathrm{C}$. A continuous closed-loop-recycling of mRNA probe solution $(0.1 \mu \mathrm{g} / \mathrm{ml}$ in hybridization $\mathrm{mix})$ was then applied for 8 hours at $70^{\circ} \mathrm{C}$. Samples were returned to room temperature and treated as follows: 30 min hybridization mix; 1 hour wash; 7 hours closed-loop-recycling of alkaline phosphatase-conjugated anti-hapten antibody (Roche; 1: 3 000); 3 hours wash; and finally, $20 \mathrm{~min}$ of Tris- $\mathrm{HCl}$ buffer $\mathrm{pH}=9.5$. The total duration of the protocol was 22 hours. Some solutions, such as probes or antibodies were collected at the end of steps for future use, others were collected for waste treatment. Enzymatic detection was then performed in NBT/BCIP solution (Roche) under constant observation.

\section{Sections and analysis}

After staining, we checked that no signal was observed with sense probes. Embryos were then fixed overnight in 4\% PFA, rinsed in PBS and cleared in an $80 \%$ glycerol solution in PBS. Embryos were photographed in whole-mounts using an Olympus SZX10 stereomicroscope equipped with DP25 color camera and Cellsens software. Embryos were rinsed for 5 min in PBS, embedded overnight in $7.5 \%$ gelatin and $15 \%$ sucrose, frozen at $-65^{\circ} \mathrm{C}$ and sectioned $(20 \mu \mathrm{m})$ using a LEICA cryomicrotome. Slides were observed under an Olympus BX41 microscope equipped with DP70 color camera and AnaliSIS software. Images were edited using Adobe Photoshop CS4 and Microsoft PowerPoint software.

\section{Acknowledgements}

We thankMaighread Gallagher-Gambarelli for advice relating to language usage and editing. Nicolas Chartier critically reviewed the manuscript. FGF8 plasmids were a kind gift from Sophie Creuzet. The ISH analysis system described in this work was funded in part by Grants-in-aid from Floralis (Grenoble, France), the Gravit innovation fund (Grenoble, France) and the Flogentec Company (Valence, France). Neither of these bodies contributed in any way to study design; data collection, analysis or interpretation; writing the report; or to the decision to submit the article for publication.

\section{References}

AL-HAKIM, A.K., GORANSSON, O., DEAK, M., TOTH, R., CAMPBELL, D.G., MORRICE, N.A., PRESCOTT, A.R. and ALESSI, D.R. (2005). 14-3-3 cooperates with LKB1 to regulate the activity and localization of QSK and SIK. J Cell Sci 118 : 5661-5673.

AMIN, N., KHAN, A., ST JOHNSTON, D., TOMLINSON, I., MARTIN, S., BRENMAN, J. and MCNEILL, H. (2009). LKB1 regulates polarity remodeling and adherens junction formation in the Drosophila eye. Proc Nat/Acad SciU SA 106: 8941-8946.

EGAN, B. and ZIERATH, J.R. (2009). Hunting for the SNARK in metabolic disease Am J Physiol Endocrinol Metab 296: E969-E972.

HAMBURGER, V. and HAMILTON, H.L. (1951). A series of normal stages in the development of the chick embryo. Dev Dyn 195: 231-272.

HARDIE, D.G. and ALESSI, D.R. (2013). LKB1 and AMPK and the cancer-metabolism link - ten years after. BMC Biol 11: 36 .

HIRANO, M., KIYONARI, H., INOUE, A., FURUSHIMA, K., MURATA, T., SUDA, Y. and AIZAWA, S. (2006). Anew serine/threonine protein kinase, Omphk1, essential to ventral body wall formation. Dev Dyn 235: 2229-2237.

HOPPE, P.E., CHAU, J., FLANAGAN, K.A., REEDY, A.R. and SCHRIEFER, L.A. (2010) Caenorhabditis elegans unc-82 encodes a serine/threonine kinase important for myosin filament organization in muscle during growth. Genetics 184: 79-90.

HOU, X., LIU, J.E., LIU, W., LIU, C.Y., LIU, Z.Y. and SUN, Z.Y. (2011). A new role of NUAK1: directly phosphorylating p53 and regulating cell proliferation. Oncogene 30: 2933-2942

KUGA, W., TSUCHIHARA, K., OGURA, T., KANEHARA, S., SAITO, M., SUZUKI, A. and ESUMI, H. (2008). Nuclear localization of SNARK; its impact on gene expression. Biochem Biophys Res Commun 377: 1062-1066.

LEFEBVRE, D.L., BAI, Y., SHAHMOLKY, N., SHARMA, M., POON, R., DRUCKER, D.J. and ROSEN, C.F. (2001). Identification and characterization of a nove sucrose-non-fermenting protein kinase/AMP-activated protein kinase-related protein kinase, SNARK. Biochem J 355: 297-305.

LU, S., NIU, N., GUO, H., TANG, J., GUO, W., LIU, Z., SHI, L., SUN, T., ZHOU, F., LI, H. et al., (2013). ARK5 promotes glioma cell invasion, and its elevated expression is correlated with poor clinical outcome. Eur J Cancer 49: 752-763.

MANNING, G., WHYTE, D.B., MARTINEZ, R., HUNTER, T. and SUDARSANAM S. (2002). The protein kinase complement of the human genome. Science 298 1912-1934.

NAMIKI, T., TANEMURA, A., VALENCIA, J.C., COELHO, S.G., PASSERON, T., KAWAGUCHI, M., VIEIRA, W.D., ISHIKAWA, M., NISHIJIMA, W., IZUMO, T. etal. (2011). AMP kinase-related kinase NUAK2 affects tumor growth, migration, and clinical outcome of human melanoma. Proc Natl Acad Sci U S A 108: 6597-6602.

OHMURA, T., SHIOI, G., HIRANO, M. and AIZAWA, S. (2012). Neural tube defects by NUAK1 and NUAK2 double mutation. Dev Dyn 241: 1350-1364.

SCOTT, J.W., ROSS, F.A., LIU, J.K. and HARDIE, D.G. (2007). Regulation of AMPactivated protein kinase by a pseudosubstrate sequence on the gamma subunit. EMBO J 26: 806-815

SUN, X., GAO, L., CHIEN, H.Y., LI, W.C. and ZHAO, J. (2013). The regulation and function of the NUAK family. J Mol Endocrinol 51: R15-22.

SUZUKI, A., KUSAKAI, G., KISHIMOTO, A., LU, J., OGURA, T., LAVIN, M.F. and ESUMI, H. (2003). Identification of a novel protein kinase mediating Akt survival signaling to the ATM protein. J Biol Chem 278: 48-53.

ULLOA, F. and MARTI, E. (2010). Wnt won the war: antagonistic role of Wnt over Shh controls dorso-ventral patterning of the vertebrate neural tube. Dev Dyn239:69-76.

VALLENIUS, T., VAAHTOMERI, K., KOVAC, B., OSICEANU, A.M., VILJANEN, M. and MAKELA, T.P. (2011). An association between NUAK2 and MRIP reveals a novel mechanism for regulation of actin stress fibers. J Cell Sci 124: 384-393.

WILKINSON, D.G. and NIETO, M.A. (1993). Detection of messenger RNA by in situ hybridization to tissue sections and whole mounts. Methods Enzymol 225:361-373. 


\section{Further Related Reading, published previously in the Int. J. Dev. Biol.}

\section{Roles of EphB3/ephrin-B1 in feather morphogenesis}

Sanong Suksaweang, Ting-Xin Jiang, Paul Roybal, Cheng-Ming Chuong and Randall Widelitz Int. J. Dev. Biol. (2012) 56: 719-728

http://dx.doi.org/10.1387/ijdb.120021rw

Skin, cornea and stem cells - an interview with Danielle Dhouailly

Cheng-Ming Chuong

Int. J. Dev. Biol. (2009) 53: 775-782

http://dx.doi.org/10.1387/ijdb.072552cc

Coexpression of Notch 3 and $R g s 5$ in the pericyte-vascular smooth muscle cell axis in response to pulp injury Henrik Lovschall, Thimios A. Mitsiadis, Knud Poulsen, Kristina H. Jensen and Annette L. Kjeldsen Int. J. Dev. Biol. (2007) 51: 715-721

http://dx.doi.org/10.1387/ijdb.072393hl

Involvement of Hex in the initiation of feather morphogenesis

Akiko Obinata and Yoshihiro Akimoto

Int. J. Dev. Biol. (2005) 49: 953-960

http://dx.doi.org/10.1387/ijdb.052079ao

The Nogent Institute - 50 Years of Embryology

Nicole Le Douarin

Int. J. Dev. Biol. (2005) 49: 85-103

http://dx.doi.org/10.1387/ijdb.041952nl

Mouse-chick neural chimeras

Josiane Fontaine-Pérus and Yvonnick Chéraud

Int. J. Dev. Biol. (2005) 49: 349-353

http://dx.doi.org/10.1387/ijdb.041943jf

Acceleration of early chick embryo morphogenesis by insulin is associated with altered expression of embryonic genes

Vidya Patwardhan, Madhavi Gokhale and Surendra Ghaskadbi

Int. J. Dev. Biol. (2004) 48: 319-326

http://dx.doi.org/10.1387/ijdb.041844vp

Entactin and laminin gamma1-chain gene expression in the early chick embryo Nikolas Zagris, Albert E. Chung and Vassilis Stavridis

Int. J. Dev. Biol. (2005) 49: 65-70

http://dx.doi.org/10.1387/ijdb.041812nz

5 yr ISI Impact Factor $(2011)=2.959$
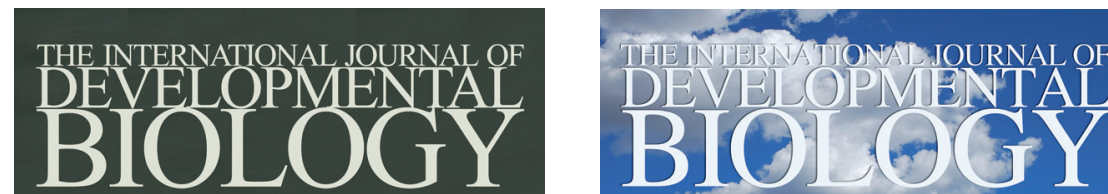

Volume 55 Nos. $4 / 5$

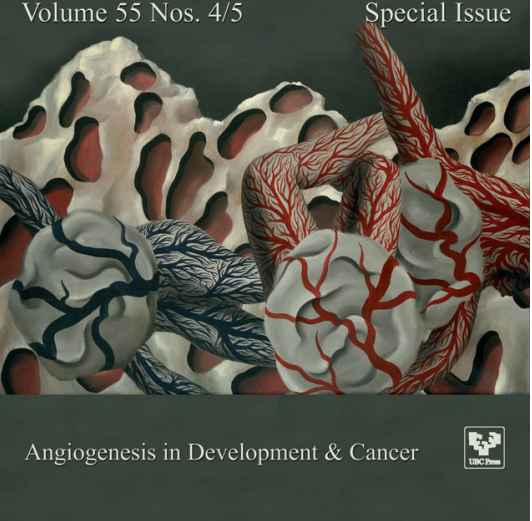

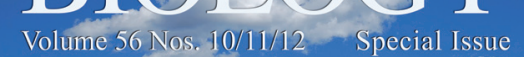

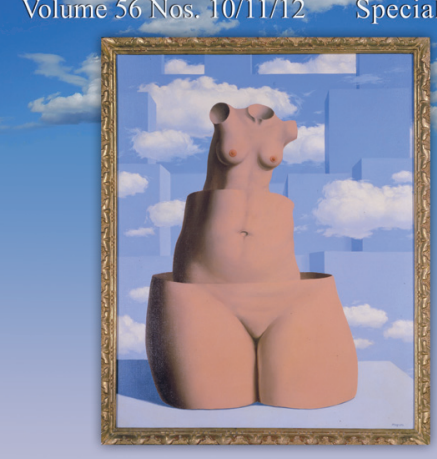

Female Germ Cells in Development \& Tumors
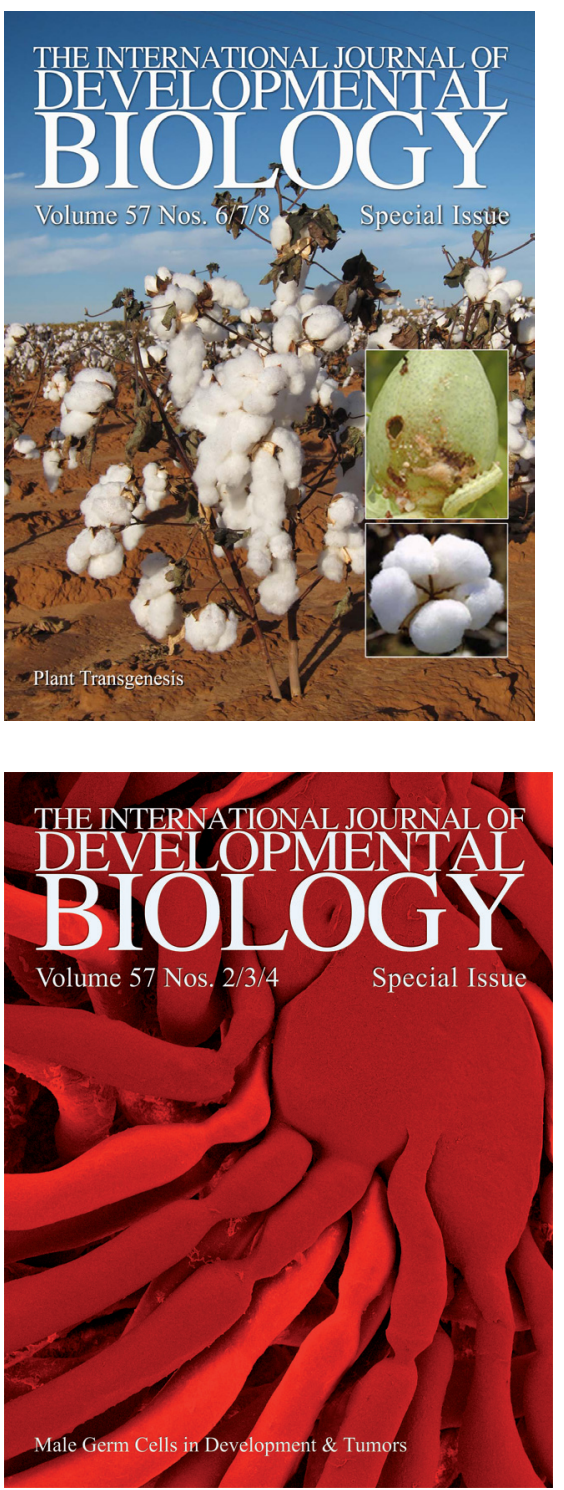